\title{
Impact of thyroid status and age on comprehensive geriatric assessment
}

\author{
Silvana Oliveira e Silva $\cdot$ I. Thien Chan $\cdot$ Maryna A. Lobo Santos • \\ Marcela Cohen - Mayra de La Roque P. Araujo - Julia da Silva Almeida • \\ Andressa Simões · Helder Renato B. Givigi • Mario Vaisman · Carlos M. Paixão Jr. • \\ Patricia de Fatima dos S. Teixeira
}

Received: 19 May 2013/ Accepted: 4 October 2013/Published online: 1 November 2013

(C) The Author(s) 2013. This article is published with open access at Springerlink.com

\begin{abstract}
This study aimed to evaluate the prevalence of thyroid dysfunction in elderly subjects attending an outpatient clinic at a tertiary hospital and to assess whether subclinical hypothyroidism (SCH) or aging affected activities of daily living (ADLs), instrumental activities of daily living (IADLs), cognitive status, or depressive symptoms. This crosssectional study included 411 patients recruited in the outpatient geriatric setting. 48 subjects reported levothyroxine use and were evaluated separately. After excluding subjects with diseases or drugs which could influence thyroid status, the 284 subjects remaining were classified as having euthyroidism $(n=235,82.8 \%)$, subclinical hypothyroidism $(n=43,15.1 \%)$, subclinical hyperthyroidism $(n=4$, $1.4 \%)$, or overt hyperthyroidism ( $n=2,0.7 \%)$. ADLs and IADLs were assessed using the Katz Index (ranging from 0 [independence] to 6 [dependence in all activities]) and Health Assessment Questionnaire (ranging from 0 to 3 [severely disabled]), respectively. Cognition was assessed using the mini mental state depressive symptoms that were
\end{abstract}

S. O. e Silva $(\bowtie) \cdot$ I. T. Chan · M. A. Lobo Santos · M. Cohen · M. de La Roque P. Araujo - J. da Silva Almeida - A. Simões · H. R. B. Givigi - M. Vaisman - P. de Fatima dos S. Teixeira Faculdade de Medicina, Universidade Federal do Rio de Janeiro, Av. Carlos Chagas Filho, 373. Edifício do Centro de Ciências da Saúde, Bloco K, Cidade Universitária, Rio de Janeiro,

RJ 21.941-902, Brazil

e-mail: medsilva@gmail.com

M. Vaisman · P. de Fatima dos S. Teixeira Endocrinology Service, Hospital Universitário Clementino Fraga Filho, Rua Professor Rodolpho Paulo Rocco, 255 Cidade Universitária, Rio de Janeiro, RJ 21941-913, Brazil

C. M. Paixão Jr.

Universidade do Estado do Rio de Janeiro, Boulevard 28 de Setembro, 87, Vila Isabel, Rio de Janeiro, RJ 20551-030, Brazil assessed using the Geriatric depression scale or cornell scale for depression in dementia. SCH did not reduce performance in ADLs or IADLs in elderly subjects as a whole, but was an independent protective factor against dependence in ADLs $(\mathrm{OR}=0.196 \quad[0.045-0.853] ; p=0.003)$ and IADLs $(\mathrm{OR}=0.060[0.010-0.361] ; p=0.002)$ in subjects aged $\geq 85$ years. Very old subjects with $\mathrm{SCH}$ showed better performance in ADLs than did those with euthyroidism (Katz Index: $0.9 \pm 1.6$ [median: 0.5] vs. $1.7 \pm 1.7$ [1.0], $p=0.024$; HAQ: $1.2 \pm 0.8$ [0.9] vs. $1.8 \pm 1.0$ [1.9], $p=0.015)$. This putative protective effect of SCH was not found in subjects aged $<85$ years. The number of falls, number of medications used, depressive symptoms, and cognitive impairment did not differ among thyroid status groups, regardless of age. In conclusion, SCH does not have impact functional performance in the elderly population as a whole, but was associated with better functional status in subjects aged $\geq 85$ years.

Keywords Activities of daily living - Thyroid function - Subclinical hypothyroidism · Katz Index · HAQ

\section{Introduction}

Thyroid hormones exert diverse effects on nervous system and overt thyroid dysfunctions are associated with several forms of cognitive impairments, peripheral neuropathies, and depressive or anxiety disorders [1]. Also, they act on muscular function [2], cardiovascular system [3, 4], erythropoiesis [5], and oxidative stress [6] among others organic functions. The implications of overt thyroid dysfunction in these systems may lead to high risk for morbid conditions and influence health related quality of life $[7,8]$ 
and mobility [9], which is an important marker of independence for activities of daily living (ALDs) in the old age.

Primary hypothyroidism is the most prevalent thyroid dysfunction in elderly individuals, and subclinical hypothyroidism, in which serum thyroid hormone levels are within normal range despite high serum thyroid-stimulating hormone (TSH) levels, is found in almost $20 \%$ of the elderly population [10-15]. Recent researches have suggested that the upper reference value for serum TSH levels varies among age strata [16-19]. However, until now, there is no exact definition of these specific cutoffs, according to age, in our population [20-22].

High serum TSH levels have been associated with exceptional longevity, with significantly higher concentrations found in centenarians than in control subjects [23].

The progression rate from subclinical to overt hypothyroidism is associated with circulating antibodies against thyroid antigens, age, and baseline serum TSH levels [20]. Subclinical hypothyroidism was recently demonstrated to persist for 4 years in slightly more than half of subjects aged $\geq 65$ years, with high rates of reversion to euthyroidism in individuals with minimally elevated serum TSH concentrations $(<7.0 \mu \mathrm{UI} / \mathrm{mL})$, who lacked circulating antibodies [24]. In the same study, a higher progression rate was not associated with subjects' age or gender [24].

Endogenous subclinical hyperthyroidism is detected in $0.5-12.4 \%$ of the general population, being more common in elderly women, especially in those living in iodine deficient areas [10-15]. The prevalence of low serum TSH in subjects taking levothyroxine is higher, reaching $30 \%$ [13]. Whether endogenous and exogenous forms have the same clinical impacts on different populations remain unclear. Subclinical hyperthyroidism is associated with a higher risk of atrial fibrillation and reduced bone mass in elderly subjects [13, 25].

Large studies have failed to demonstrate an association between cognitive dysfunction and subclinical hypo or hyperthyroidism in elderly subjects [26, 27]. However, the results of the Invecchiare in Chianti Study [28] showed that abnormal cognitive function, detected by mini mental state examination (MMSE) scores less them 24 points, was more commonly associated with subclinical hyperthyroidism diagnosis [28]. In contrast, small studies applying very sensitive tests to evaluate specific cognitive domains have shown subtle changes in some cognitive areas (e.g., memory and executive function), but the clinical relevance of these results remains unknown [29-31].

Subclinical hypothyroidism was not found to be associated with dependence in ADLs, depressive symptoms, or cognitive function in a cohort of 599 subjects aged 85-89 years [32]. Furthermore, subjects from the same group, with mild elevations of serum $\mathrm{TSH}$, had better walking speeds, and cardiopulmonary performance compared with those without thyroid dysfunction. Slightly elevated serum TSH levels were also associated with better global survive in another cohort, regardless of serum free triiodothyronine (FT3) levels, cognitive function, or global functional performance [32].

These findings have being considered in clinical practice to suggest that patients with minimal elevations of serum TSH should not be treated when their age are above a specific range [20-22, 33, 34]. A doubt is that regardless to possible improve quality of life of elderly subjects the restoration of euthyroidism might be associated with worse endpoints [20-22].

Recently, a differentiation in the approach of subclinical hypothyroidism in older people has been proposed, suggesting that L-T4 replacement should be avoid in the oldest old subjects, but should be considered in the modest old one, according to their cardiovascular risk [33]. If the impact of slightly elevations of serum TSH in ADLs, cognitive status, or depressive symptoms of elderly subjects will differ according to specific age grade, it is unknown yet.

The aim of the present study was to evaluate the prevalence of thyroid dysfunction in elderly subjects attending an outpatient clinic at a tertiary hospital. We also assessed whether thyroid function, according to different aging grade impacted instrumental activities of daily living (IADLs), ADLs, cognitive status, or depressive symptoms.

\section{Patients and methods}

\section{Study design and population}

Participants in this cross-sectional study were recruited from the outpatient geriatric clinic of Clementino Fraga Filho University Hospital, Federal University of Rio de Janeiro, between 2010 and 2012. All subjects $\geq 65$ years old, who regularly attended the clinic were considered for inclusion. The institution's ethics committee approved the study, and all patients provided written informed consent.

\section{Exclusion criteria}

We excluded subjects with recent hospitalization $(n=39)$, recent radioiodine use $(n=16)$, and histories of amiodarone use $(n=21)$. Three individuals with serum free thyroxine (FT4) levels below the reference range, and inappropriately normal serum TSH levels were also excluded.

Clinical evaluation

All included subjects were evaluated clinically via specific interviews, and all medical records were reviewed by one of the authors. The following data were collected: gender, 
age, anthropometric measures (body mass index, BMI obtained by direct or indirect measures) [34], recent hospitalizations, falls in the last year, comorbidities (diabetes mellitus, high blood pressure, history of coronary heart disease, heart failure [confirmed by echocardiographic evaluation], or cerebrovascular disease), history of thyroid disease or radioiodine use, tobacco use, and current medical prescriptions. Coronary heart disease was considered present when the patient had a past history of hospital admission for instable angina or myocardial infarct or even if the patient had a positive investigation for stable angina (invasive or noninvasive). Only past stroke history was considered as positive cerebrovascular disease present.

Functional evaluation

Functional status was assessed using well-known scales adapted for and validated in Brazilian populations. ADLs (bathing, dressing, toileting, transferring, continence, and feeding) were assessed using the Katz Index [35-37]; a sixitem interview which scores are ranging from 0 (independence in all six activities) to 6 (dependence in all activities). Any grade of dependence (scores $\geq 1$ ) was considered as abnormal Katz Index. IADLs were assessed using the Brazilian version of the Health Assessment Questionnaire (HAQ) disability index [38-40]. The HAQ was originally designed to evaluate adults with arthritis, but it has been used to evaluate health in a wide range of research settings. It is based on a hierarchical model that considers disease effects in terms of death, disability, discomfort, side effects of treatment, and medical costs. Only the disability dimension, which comprises 20 questions about daily functioning during the past week, was used in the present study. These items are classified into eight components: dressing and grooming, arising, reaching, eating, gripping, walking, hygiene, and outdoor activities. Scores are ranging from 0 (completely self-sufficient) to 3 (severely disabled) [41]. Abnormal HAQ indexes were considered when the punctuations scored $>1$.

Cognition was assessed using the MMSE [42]. The cutoff value for cognitive impairment was modulated according to educational level for the Brazilian population, as suggested by Lourenço and Veras [43]. They proposed cutoff values of $24 / 30$ for those with formal education (1 year at school and more) and 18/30 for those without.

Depressive symptoms were assessed using two instruments, according to cognition. For subjects with MMSE scores $\geq 13$, we used the 15 -item version of the Geriatric depression scale (GDS) [44, 45]. Scores $\geq 6 / 15$ were considered to be suggestive of depressive disorder. For subjects with MMSE scores $<13$, the Cornell scale for depression in dementia (CSDD) was used [46, 47], with scores $\geq 10$ indicating probable depressive disorder.
Laboratory tests

Blood samples were collected at morning, and tested to determine TSH, FT4, and anti-thyroperoxidase antibody (TPO-Ab) levels using a third-generation chemiluminescence kit (Immulite $2000^{\circledR}$; Diagnostic Products Corporation). The respective reference ranges were $0.4-4.0 \mu \mathrm{UI} /$ $\mathrm{mL}, 0.8-1.9 \mathrm{ng} / \mathrm{dL}$, and $<35 \mathrm{UI} / \mathrm{mL}$.

Age and thyroid status classification

The study population was stratified into two age subgroups: old ( $<85$ years) and very old ( $\geq 85$ years). Subjects were also divided into five groups, according to thyroid status: (1) euthyroidism (TSH level within reference range), (2) subclinical hypothyroidism (TSH level >4.0-19.9 $\mu \mathrm{UI} / \mathrm{mL}$, FT4 level normal), (3) overt hypothyroidism (TSH level $\geq 20$, FT4 level below reference range), (4) overt hyperthyroidism, (TSH level $<0.4 \mu \mathrm{UI} / \mathrm{mL}$, FT4 level above reference range), and (5) subclinical hyperthyroidism (TSH level $<0.4 \mu \mathrm{UI} / \mathrm{mL}$, FT4 level normal).

Statistical analyses

All analyses were performed using SPSS software (ver. 13.0 for Windows; SPSS Inc., Chicago, IL, USA). Continuous variables were described as means \pm standard deviations (medians) and compared between/among groups using Student's $t$ test or the Mann-Whitney test. Score punctuations for functional scales were assessed as continuous and categorical variables. The Kolmogorov-Smirnov test with Lilliefors correction was used to assess the Gaussian nature of pattern distributions. Proportions were compared using the Chi squared or Fisher's exact test. Spearman correlation analyses were performed between serum TSH level and eight variables (age, BMI, number of falls in the past year, and Katz Index, HAQ, MMSE, GDS, and CSDD scores). Correlation values $\leq 0.20$ were considered significant.

Binary logistic regression analysis was performed with abnormal IADL and ADL scores serving as dependent variables and confounding variables included in multivariate analysis (very old age [ $\geq 85$ years], subclinical hypothyroidism, coronary artery disease, heart failure, cerebrovascular disease, depressive disorder, cognitive disorder, and $\geq 3$ falls in the last year). All variables were included in each step model by entry method. A significance level of $5 \%$ was used. Binary logistic regression analysis stratified by age group was also performed, including all previously mentioned variables, except very old age.

\section{Results}

Of 563 patients recruited in the outpatient geriatric setting, 411 (316 women and 95 men) provided informed consent 
Fig. 1 Population samples distribution in the study

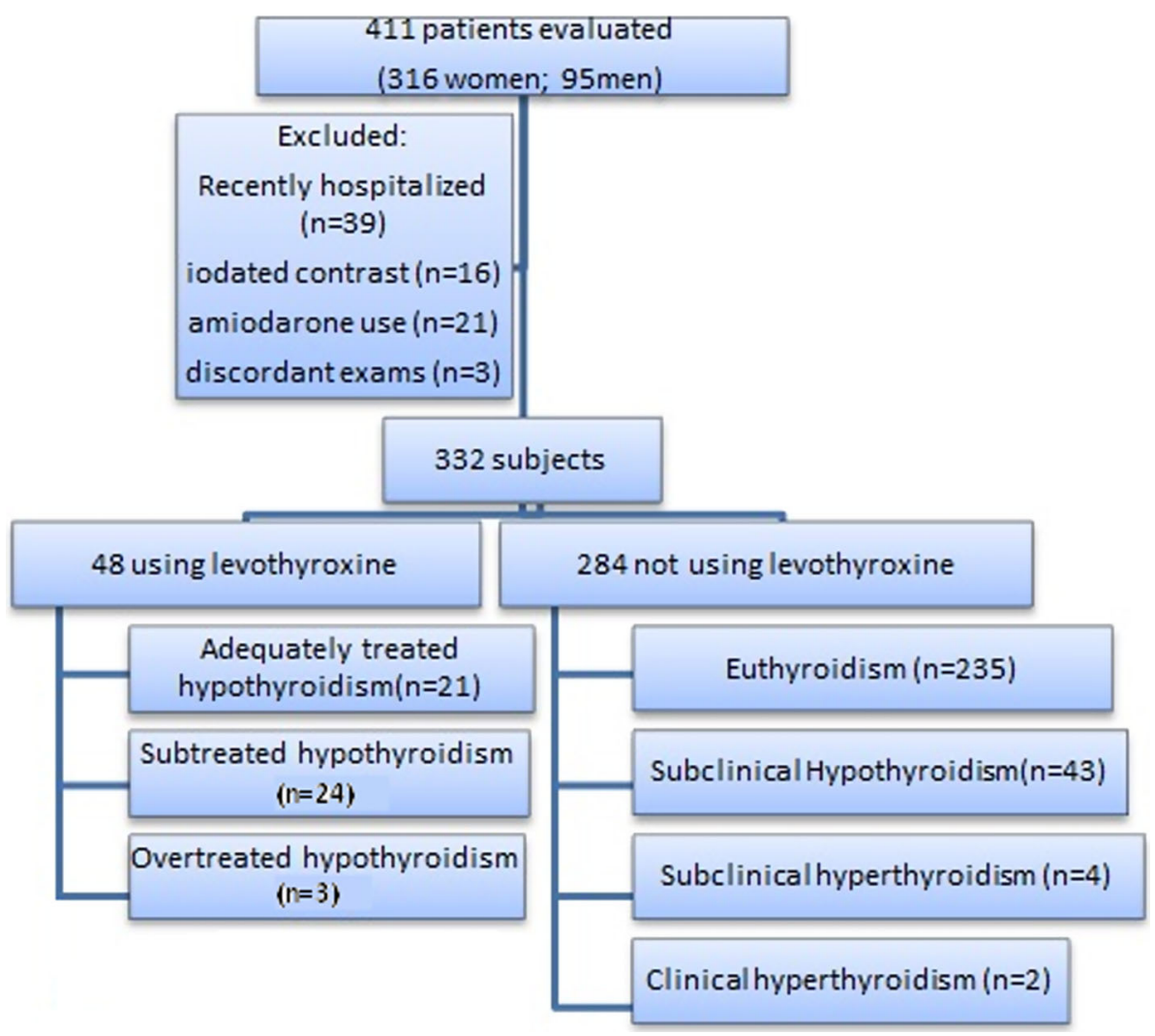

and were included in the study. 48 subjects reported levothyroxine use and were evaluated separately. Exclusion criteria described previously were then applied (e.g., amiodarone or iodine contrast use and recent hospitalization). The remaining 284 individuals were categorized as having euthyroidism ( $n=235,82.8 \%$ ), subclinical hypothyroidism $(n=43,15.1 \%)$, subclinical hyperthyroidism $(n=4$, $1.4 \%)$, or overt hypothyroidism $(n=2,0.7 \%)$. Patients with overt hypothyroidism or subclinical hyperthyroidism were not submitted to further evaluation because of the small numbers of individuals in these groups. No case of overt hypothyroidism without hormone replacement was detected. The remaining 278 subjects had a mean age of $80.7 \pm 67$ years (range 65-102); 203 subjects were aged $<85$ years, and 75 were aged $\geq 85$ years (Fig. 1 ).

Patients using levothyroxine

After the initial application of exclusion criteria, we evaluated, apart from the whole group, 48 patients who were using levothyroxine at the time of evaluation (Fig. 1). They were divided into three subgroups: adequately treated (TSH within the reference range), undertreated ( $\mathrm{TSH}>4.0 \mu \mathrm{UI} /$ $\mathrm{mL}$ ), and overtreated ( $\mathrm{TSH}<0.4 \mu \mathrm{UI} / \mathrm{mL}$ ) hypothyroidism. About half $(n=24)$ of these patients were adequately treated, $6.0 \%(n=3)$ were taking excessive levothyroxine doses, and $44.0 \%(n=21)$ were undertreated.
Undertreated subjects tended to have higher Katz Index scores $(2.1 \pm 1.9$ [2.0]) than adequately treated $(1.3 \pm 1.3$ [1.0]) and overtreated (1.7 \pm 1.2 [1.0]) subjects, but this difference was not significant. Similarly, undertreated subjects tended to have higher HAQ scores than adequately and overtreated subjects $(1.9 \pm 0.9$ [2.0] vs. $1.4 \pm 0.8$ [1.4] and $1.1 \pm 1.2[0.8]$, respectively; both $p>0.10$ ). Adequately treated subjects' performance in ADLs and IADLs was similar to that of subjects with euthyroidism and no levothyroxine replacement (Katz Index: $1.3 \pm 1.3$ [1.0] vs. $1.2 \pm 1.6[1.0], p=0.21$ and HAQ index: $1.4 \pm 0.8[1.4]$ vs. $1.4 \pm 0.9[1.4], p=0.431)$. No significant difference among the three treated subgroups was found in any other variable (BMI, MMSE score, total number of medications in use, gender, $\mathrm{TPO}-\mathrm{Ab}+$, recurrent falls, the presence of cognitive deficit, depressive symptoms, cerebrovascular or coronary artery disease, and heart failure [data not shown]).

Patients without levothyroxine replacement

General and functional characteristics of the study groups are shown in Table 1, according to thyroid status, including those without levothyroxine replacement. Demographic, functional, and risk-factor variables did not differ among subjects with euthyroidism and those with subclinical hypothyroidism. 
Table 1 Group characteristics by thyroid status

\begin{tabular}{|c|c|c|c|c|c|}
\hline & \multicolumn{3}{|c|}{ Subjects undergoing levothyroxine replacement $(n=48)$} & \multicolumn{2}{|c|}{$\begin{array}{l}\text { Subjects with no condition influencing } \\
\text { thyroid function }(n=278)\end{array}$} \\
\hline & Undertreated & $\begin{array}{l}\text { Adequately } \\
\text { treated }\end{array}$ & Overtreated & Euthyroidism & $\begin{array}{l}\text { Subclinical } \\
\text { hypothyroidism }\end{array}$ \\
\hline$n(\%)$ & $21(44.0)$ & $24(50.0)$ & $3(6.0)$ & $235(84.5)$ & $43(15.4)$ \\
\hline Age (years) & $\begin{array}{l}82.7 \pm 6.8 \\
\quad(82.0)\end{array}$ & $79.9 \pm 5.8(81.0)$ & $81.3 \pm 8.7(79.0)$ & $\begin{array}{l}80.3 \pm 6.8 \\
\quad(80.0)\end{array}$ & $81.2 \pm 6.7(83.0)$ \\
\hline Women $(\%)$ & 85.7 & 83.7 & 66.7 & 76.6 & 81.4 \\
\hline $\mathrm{BMI}\left(\mathrm{kg} / \mathrm{m}^{2}\right)$ & $\begin{array}{l}33.9 \pm 3.7 \\
(26.6)\end{array}$ & $30.9 \pm 5.8(30.9)$ & $27.5 \pm 5.3(26.4)$ & $\begin{array}{l}27.2 \pm 4.7 \\
\quad(26.6)\end{array}$ & $27.0 \pm 4.5(27.7)$ \\
\hline $\mathrm{TSH}(\mu \mathrm{UI} / \mathrm{mL})$ & $6.0 \pm 3.0(5.5)^{*}$ & $2,1 \pm 1.2(1.8)^{*}$ & $\begin{array}{l}0.03 \pm 0.05 \\
(0.0)^{*}\end{array}$ & $2.1 \pm 0.9(2.0)^{*}$ & $5.5 \pm 1.2(5.1)^{*}$ \\
\hline FT4 (ng/dL) & $1.1 \pm 0.2(1.1)$ & $1.2 \pm 0.2(1.2)^{\#}$ & $1.6 \pm 0.3(1.5)^{\#}$ & $1.1 \pm 0.2(1.1)$ & $1.1 \pm 0.14(1.0)$ \\
\hline $\mathrm{TPO}-\mathrm{Ab}+(\%)$ & 53.0 & 52.0 & 0.0 & 12.2 & 10.5 \\
\hline Medications in use $(n)$ & $8.2 \pm 2.7(7.0)$ & $8.5 \pm 2.6(8.0)$ & $7.9 \pm 2.7(7.0)$ & $6.4 \pm 2.8$ & $6.8 \pm 2.8$ \\
\hline$\geq 3$ falls in the last year & 14.3 & 4.2 & 0 & 11.6 & 9.3 \\
\hline Katz Index & $2.1 \pm 1.9(2.0)$ & $1.3 \pm 1.3(1.0)$ & $1.7 \pm 1.2(1.0)$ & $1.2 \pm 1.6(1.0)$ & $1.2 \pm 1.7(1.0)$ \\
\hline $\begin{array}{l}\text { Functional dependence-Katz Index } \\
(\%)\end{array}$ & 81.0 & 78.3 & 100 & 57.4 & 60.5 \\
\hline HAQ & $1.9 \pm 0.9(2.0)$ & $1.4 \pm 0.8(1.4)$ & $1.1 \pm 1.2(0.8)$ & $1.4 \pm 0.9(1.4)$ & $1.4 \pm 0.9(1.4)$ \\
\hline Functional dependence- HAQ (\%) & 71.4 & 69.6 & 33.3 & 59.1 & 55.8 \\
\hline MMSE & $19.7 \pm 7.3(22.0)$ & $22.1 \pm 7.6(24.0)$ & $20.0 \pm 7.2(21.5)$ & $\begin{array}{l}19.3 \pm 8.2 \\
(20.0)\end{array}$ & $19.7 \pm 7.9(20.0)$ \\
\hline Cognitive deficit $(\%)$ & 57.1 & 45.8 & 33.3 & 54.1 & 60.5 \\
\hline Depressive disorder $(\%)$ & 57.1 & 54.2 & 0.0 & 45.6 & 52.4 \\
\hline $\mathrm{CAD}(\%)$ & 5.6 & 8.3 & 33.3 & 9.4 & 2.4 \\
\hline Heart failure $(\%)$ & 22.2 & 16.0 & 0.0 & 32.8 & 35.7 \\
\hline CVD (\%) & 25 & 21.7 & 27.8 & 15.9 & 21.4 \\
\hline
\end{tabular}

Values are presented as $n(\%)$ or mean \pm standard deviation (median)

$S D$ standard deviation, $B M I$ body mass index, TSH thyroid-stimulating hormone, FT4 free thyroxine, TPO-AB anti-thyroperoxidase antibody, $H A Q$ health assessment questionnaire, $C A D$ coronary artery disease, $C V D$ cerebrovascular disease

${ }^{*} p<0.001$ comparing subjects with euthyroidism and SHypo, and also comparing three treat groups; ${ }^{*} p<0.001$ comparing overtreated and adequately treated subjects; No other significant difference was observed between subjects with SHypo and euthyroidism, among treated groups, or between those with euthyroidism and adequately treated subjects

ADL and IADL scores are shown in Table 2 according to age group, independent of thyroid status. As expected, ADL and IADL scores were significantly higher among very old subjects than among old individuals (Katz Index: $1.6 \pm 1.7[1.0]$ vs. $1.1 \pm 1.6[1.0], p=0.001 ;$ HAQ: $1.7 \pm 0.8[1.6]$ vs. $1.3 \pm 0.9[1.1], p<0.001)$ and very old subjects had more cognitive impairment (71.6 vs. $49.0 \%, p=0.001)$.

Results of multivariate analyses are shown in Tables 3 and 4. In the group as a whole, the risk of dependence in ADLs, as measured by the Katz Index, was independently related to cognitive impairment (odds ratio, $\mathrm{OR}=2.555,95 \%$ confidence interval, CI $1.506-4.334 ; p=0.001)$ and very old age $(\mathrm{OR}=2.381,95 \%$ CI 1.249-4.538; $p=0.008)$, but was not related to the presence of subclinical hypothyroidism $(\mathrm{OR}=0.765,95 \%$ CI 0.369-1.899). The risk of abnormal
HAQ score was independently related to very old age $(\mathrm{OR}=2.816,95 \%$ CI $1.451-45.468 ; p=0.002)$, depressive disorder $(\mathrm{OR}=1.830,95 \%$ CI $1.063-3.150$; $p=0.029)$, and cognitive impairment $(\mathrm{OR}=2.422,95 \%$ CI $1.413-4.154 ; p=0.001)$. Evaluating the whole group, subclinical hypothyroidism did not reduce elderly subjects' performance in ADLs or IADLs (Fig. 2).

Impact of thyroid status on functional status of patients without levothyroxine replacement, stratified by age

ADL scores were lower in very old subjects with subclinical hypothyroidism than in those with euthyroidism (Katz Index: $0.9 \pm 1.6[0.5]$ vs. $1.7 \pm 1.7[1.0], p=0.024$; HAQ: $1.2 \pm 0.8$ [0.9] vs. $1.8 \pm 0.8[1.9], p=0.015$; Table 5). This putative protective effect of subclinical hypothyroidism 
Table 2 Subjects characteristics by age group

\begin{tabular}{|c|c|c|}
\hline Variable & $<85$ years & $\geq 85$ years \\
\hline$n(\%)$ & $203(73)$ & $75(27)$ \\
\hline Age (years) & $\begin{array}{l}77.4 \pm 4.8 \\
\quad(78)^{*}\end{array}$ & $\begin{array}{l}88.6 \pm 3.7 \\
(88.0)^{*}\end{array}$ \\
\hline Women $(\%)$ & 76.8 & 78.7 \\
\hline BMI $\left(\mathrm{kg} / \mathrm{m}^{2}\right)$ & $27.6 \pm(27.2)^{\dagger}$ & $26.1 \pm(26.2)^{\dagger}$ \\
\hline TSH $(\mu \mathrm{UI} / \mathrm{mL})$ & $\begin{array}{l}2.5 \pm 1.5 \\
(2.1)\end{array}$ & $2.9 \pm 1.7(2.6)$ \\
\hline FT4 $(\mu \mathrm{UI} / \mathrm{mL})$ & $\begin{array}{l}1.1 \pm 0.1 \\
(1.1)\end{array}$ & $1.2 \pm 0.2(1.1)$ \\
\hline $\mathrm{TPO}-\mathrm{Ab}+(\%)$ & 11.4 & 13.3 \\
\hline Falls in last year & $\begin{array}{l}1.2 \pm 1.9 \\
(1.0)\end{array}$ & $0.9 \pm 1.3(0.0)$ \\
\hline Medications used & $\begin{array}{l}6.6 \pm 2.7 \\
(7.0)\end{array}$ & $6.2 \pm 2.8(6.0)$ \\
\hline Katz Index & $\begin{array}{c}1.1 \pm 1.6 \\
(1.0)^{* *}\end{array}$ & $\begin{array}{l}1.6 \pm 1.7 \\
(1.0)^{* *}\end{array}$ \\
\hline $\begin{array}{l}\text { Functional dependence, Katz } \\
\text { Index }(\%)\end{array}$ & $51.7 * *$ & $74.7 * *$ \\
\hline HAQ & $\begin{array}{l}1.3 \pm 0.9 \\
(1.1)^{*}\end{array}$ & $\begin{array}{l}1.7 \pm 0.8 \\
(1.6)^{*}\end{array}$ \\
\hline Functional dependence, HAQ (\%) & $52.2 * * *$ & $76.0 * * *$ \\
\hline Cognitive deficit (\%) & $49.0 * *$ & $71.6^{* *}$ \\
\hline Depressive symptoms (\%) & 44.2 & 53.5 \\
\hline CVD $(\%)$ & 17.0 & 16.2 \\
\hline Heart failure $(\%)$ & 31.0 & 39.2 \\
\hline CAD $(\%)$ & 7.5 & 10.8 \\
\hline
\end{tabular}

Continuous variables are presented as mean \pm standard deviation (median)

$B M I$ body mass index, TSH thyroid-stimulating hormone, FT4 free thyroxine, TPO-Ab anti-thyroperoxidase antibody, $H A Q$ health assessment questionnaire, $C V D$ cerebrovascular disease, $C A D$ coronary artery disease

$* p=0.000 ; * * p=0.001 ; * * * p<0.001 ;{ }^{\dagger} p=0.023$ was not found in subjects aged $<85$ years (Fig. 3). The number of falls, number of medications used, depressive symptoms, and cognitive impairment did not differ among thyroid status groups, regardless of age.

Age-stratified analysis revealed a weak positive correlation between serum TSH level and MMSE score in patients aged $<85$ years ( $r s=0.144, p=0.021$ ), but no correlation between TSH level and any other variable in this subgroup (data not shown). In the very old group, no correlation was found between serum TSH level and any other studied variable (data not shown).

In patients aged $<85$ years, only cognitive deficit was an independent risk factor for dependence in ADLs, assessed by the Katz Index $(\mathrm{OR}=2.733,95 \%$ CI 1.486-5.029; $p=0.001)$. The risk of dependence in IADLs, assessed by the HAQ, was independently related to cognitive deficit and the presence of a depressive disorder (Tables 3, 4). Subclinical hypothyroidism did not influence the risk of dependence in ADLs or IADLs (Tables 3, 4; Fig. 2). In the very old group, subclinical hypothyroidism was an independent protective factor related to dependence in ADLs $(\mathrm{OR}=0.196,95 \%$ CI $0.045-0.853 ; p=0.003)$ and IADLs $(\mathrm{OR}=0.060$, $95 \%$ CI $0.010-0.361 ; p=0.002$; Tables 3, 4; Fig. 2).

\section{Discussion}

Our study findings indicate that thyroid status has no impact on functional performance in the elderly population as a whole, but patients aged $<85$ years cannot be evaluated in the same manner as very old subjects. Measurement of the impact of thyroid function in elderly individuals using different endpoints according to age has recently been proposed [15, 20-22, 33, 48, 49]. The results of the

Table 3 Variables independently associated with a high risk for functional dependence, measured by the Katz Index

\begin{tabular}{|c|c|c|c|c|c|c|}
\hline & \multirow[t]{2}{*}{ Beta coefficient } & \multirow[t]{2}{*}{ Standard error } & \multirow[t]{2}{*}{$p$} & \multirow[t]{2}{*}{ OR } & \multicolumn{2}{|c|}{$95 \% \mathrm{CI}$} \\
\hline & & & & & Lower & Upper \\
\hline \multicolumn{7}{|l|}{ Whole group } \\
\hline Cognitive deficit ${ }^{\mathrm{a}}$ & 0.938 & 0.270 & 0.001 & 2.555 & 1.506 & 4.334 \\
\hline Very old age ${ }^{b}$ & 0.867 & 0.329 & 0.008 & 2.381 & 1.249 & 4.538 \\
\hline \multicolumn{7}{|l|}{ Old ( $<85$ years) } \\
\hline Cognitive deficit ${ }^{\mathrm{b}}$ & 1.005 & 0.311 & 0.001 & 2.733 & 1.486 & 5.029 \\
\hline \multicolumn{7}{|l|}{ Very old ( $\geq 85$ years) } \\
\hline Subclinical hypothyroidism ${ }^{\mathrm{b}}$ & -1.630 & 0.750 & 0.030 & 0.196 & 0.045 & 0.853 \\
\hline
\end{tabular}


Table 4 Variables independently associated with a high risk for functional dependence, measured by the HAQ

\begin{tabular}{|c|c|c|c|c|c|c|}
\hline & \multirow[t]{2}{*}{ Beta coefficient } & \multirow[t]{2}{*}{ Standard error } & \multirow[t]{2}{*}{$p$} & \multirow[t]{2}{*}{ OR } & \multicolumn{2}{|c|}{$95 \% \mathrm{CI}$} \\
\hline & & & & & Lower & Upper \\
\hline \multicolumn{7}{|l|}{ Whole group ${ }^{\mathrm{a}}$} \\
\hline Cognitive Impairment & 0.885 & 0.329 & 0.001 & 2.422 & 1.413 & 4.154 \\
\hline Very old age & 1.035 & 0.338 & 0.002 & 2.816 & 1.451 & 5.468 \\
\hline Depressive disorder & 0.604 & 0.456 & 0.029 & 1.830 & 1.063 & 3.150 \\
\hline \multicolumn{7}{|l|}{ Old $(<85 \text { years })^{\mathrm{b}}$} \\
\hline Cognitive deficit & 1.024 & 0.314 & 0.001 & 2.785 & 1.504 & 5.158 \\
\hline Depressive disorder & 0.836 & 0.319 & 0.009 & 2.307 & 1.235 & 4.308 \\
\hline \multicolumn{7}{|l|}{ Very old $(\geq 85 \text { years })^{b}$} \\
\hline Subclinical hypothyroidism & -2.813 & 0.916 & 0.002 & 0.060 & 0.010 & 0.361 \\
\hline
\end{tabular}

OR odds ratio, $C I$ confidence interval

a Binary logistic regression model adjusted for depressive disorder, cognitive deficit, the presence of subclinical hypothyroidism, coronary artery disease, heart failure, recurrent falls, very old age, and cerebrovascular disease

b Binary logistic regression model adjust for all factors listed above except very old age

Fig. 2 Adjusted for the presence of cognitive defect, depressive disorder, coronary artery disease, cerebrovascular disease, heart failure, and recurrent falls. asterisk indicates additional adjust for very old age
Subclinical Hypothyroidism and abnormal KATZ index of elderly subjects

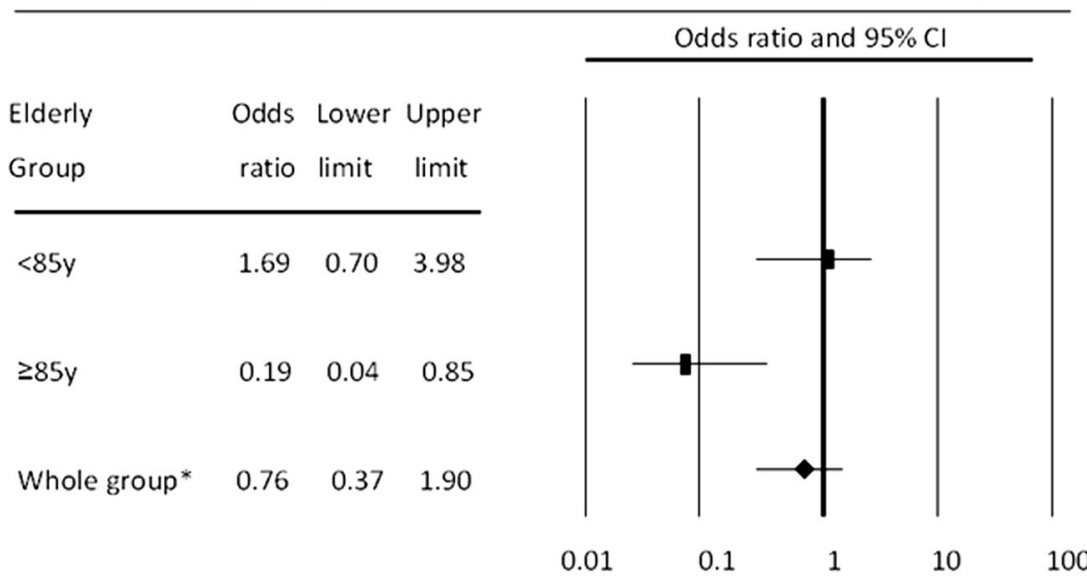

Subclinical Hypothyroidism and abnormal HAQ index of elderly subjects

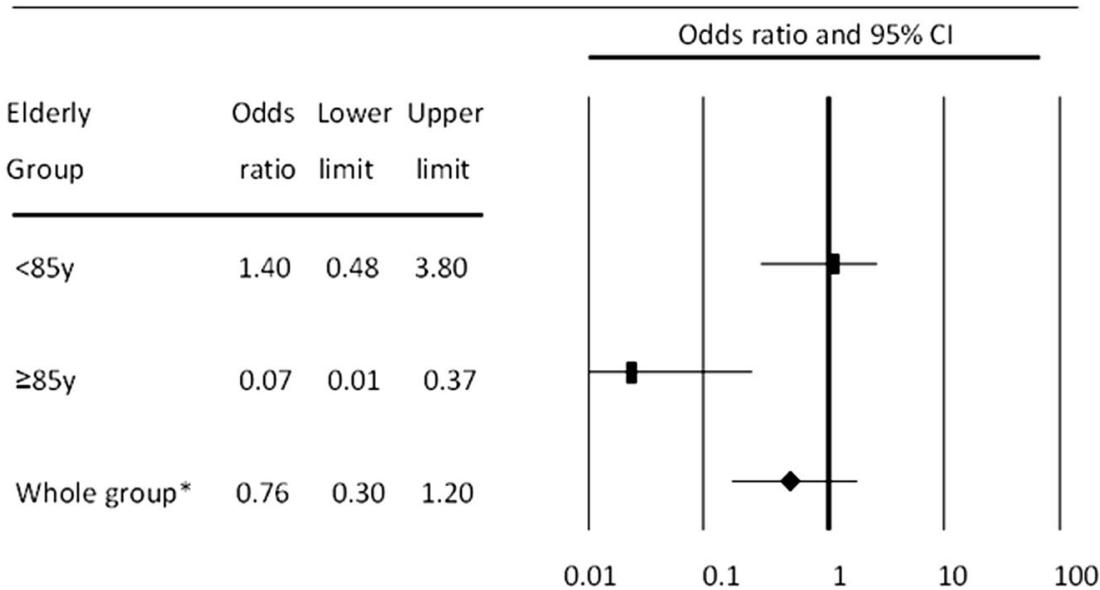

more comorbidities and worse functional performance than those aged $<85$ years, these very old subjects when presented with serum TSH levels above the reference range hypothyroidism on performance in ADLs and IADLs very elderly people. Although subjects aged $\geq 85$ years had 
Table 5 Functional profiles in subjects with euthyroidism and subclinical hypothyroidism, by age group

\begin{tabular}{|c|c|c|c|c|}
\hline \multirow{2}{*}{$\begin{array}{l}\text { Variable } \\
\text { Thyroid status }\end{array}$} & \multicolumn{2}{|l|}{$<85$ years } & \multicolumn{2}{|l|}{$\geq 85$ years } \\
\hline & Euthyroidism & Subclinical hypothyroidism & Euthyroidism & Subclinical hypothyroidism \\
\hline$n$ & 174 & 29 & 61 & 14 \\
\hline Katz Index & $1.00 \pm 1.5(0.0)$ & $1.4 \pm 1.7(1.0)$ & $1.7 \pm 1.7(1.0)^{*}$ & $0.9 \pm 1.6(0.5)^{*}$ \\
\hline Functional dependence, Katz Index (\%) & 49.4 & 65.5 & $80.3^{\dagger}$ & $50.0^{\dagger}$ \\
\hline HAQ & $1.2 \pm 0.9(1.1)$ & $1.5 \pm 1.0(1.5)$ & $1.8 \pm 0.8(1.9)^{* *}$ & $1.2 \pm 0.8(0.9)^{* *}$ \\
\hline Functional dependence, HAQ (\%) & 44.8 & 62.1 & $78.7^{\alpha}$ & $35.7^{\alpha}$ \\
\hline Recurrent falls $(\%)^{\mathrm{a}}$ & 11.0 & 10.3 & 13.6 & 7.1 \\
\hline Medications used & $6.5 \pm 2.7(6.0)$ & $7.4 \pm 2.6(7.0)$ & $6.4 \pm 2.9(6.0)$ & $5.4 \pm 2.6(5.5)$ \\
\hline Cognitive deficit (\%) & 48.6 & 51.7 & 70.0 & 78.6 \\
\hline Heart failure & 29.7 & 39.3 & 41.7 & 29.0 \\
\hline Coronary artery disease & 8.1 & 3.6 & 13.3 & 0.0 \\
\hline Cerebrovascular disease & 15.1 & 28.6 & 18.3 & 7.1 \\
\hline Depressive disorder (\%) & 44.4 & 42.9 & 49.1 & 71.4 \\
\hline
\end{tabular}

Continuous variables are presented as mean \pm standard deviation (median)

SHypo subclinical hypothyroidism, $H A Q$ health assessment questionnaire

$* p=0.024 ; * * p=0.015 ;{ }^{\dagger} p=0.036 ;{ }^{\alpha} p=0.004$

a Three or more falls in the last year

\section{IADL Dependence in Elderly and Very Elderly Subjects, HAQ}

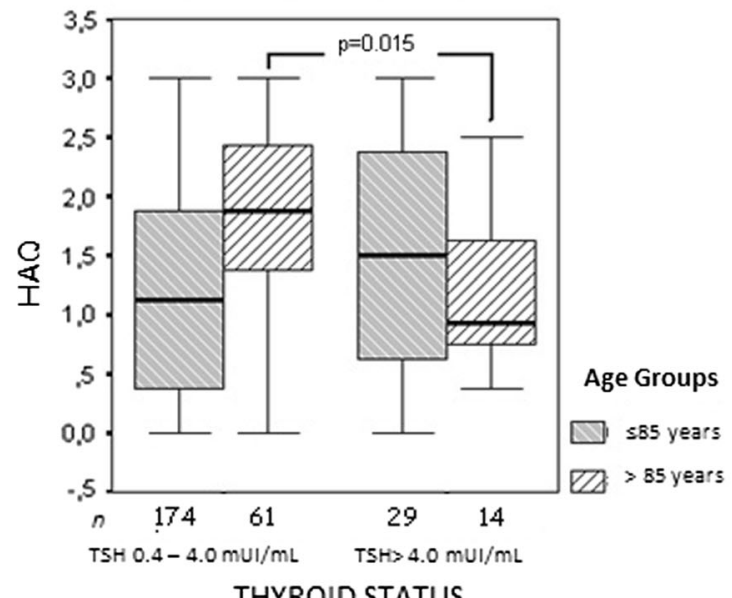

THYROID STATUS

\section{ADL Dependence in Elderly and Very Elderly Subjects, Katz Index}

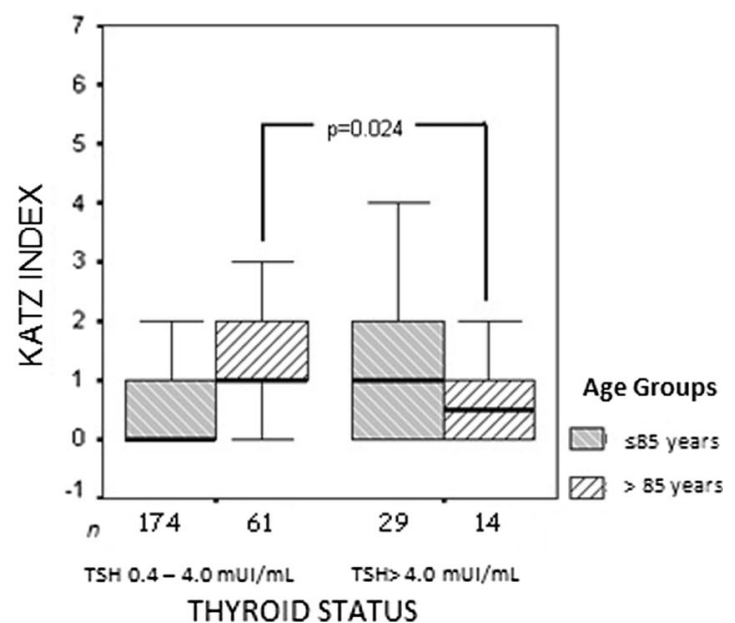

Fig. 3 Comparisons between HAQ e Katz Indexes from elderly patients with SHypo versus "normal" serum TSH according to different age subgroups

showed better performance in ADLs and IADLs than euthyroid very old patients in same age group. These results are in accordance with previously published data on mobility in elderly individuals [9, 32]. Several studies have presented evidence supporting a change in the distribution of the serum TSH normality curve according to age, with a shift to higher values for very old individuals [16, 17, 49, 50]. This change, underlain by genetic factors, modifications in central set-point for thyroid hormone feed-back, and dietary iodine supply, would provide better guidance for further evaluation of thyroid dysfunction and would reflect growing support for the assumption that minimally elevated serum TSH levels in elderly subjects should be considered normal [16, 17, 49, 50]. Very old individuals with high thyrotropin levels do not experience adverse effects or have higher mortality rates, and thus may not require levothyroxine replacement [21-23, 32].

Few large studies have examined associations between functionality and thyroid function [9, 32]. More such studies are necessary, given the evidence for an association between a subtle reduction in thyroid activity and better functionality in elderly individuals. 
Comorbidities were prevalent, and the risks of frailty and functional decline were high in the population of the present study, which was recruited in an outpatient setting. Few studies have examined thyroid status in similar populations due to the difficulties presented by the prevalence of adverse endpoints and the high risk of follow-up interruption. Thus, the results of the present study should be compared with data from populations with similar characteristics.

As expected, dependence in ADLs and IADLs and cognitive decline were more prevalent in very old than in old subjects, reflecting a global decline related to aging [50-52]. We also found lower BMIs in very old people than in the old group. Both age strata are eutrophic, but these lower values may be associated with changes in body composition, muscle loss, and frailty in very old subjects; additional research is needed to define the associations between body composition and thyroid function in old and very old subjects. Despite these results, functional performance differed according to serum TSH level in very old subjects, as reported above.

The prevalence of thyroid disease in this study was similar to that reported in previous studies, although the prevalence of subclinical hypothyroidism was almost three-fold higher than that found in a study of communitybased elderly Brazilians [18].

Since almost $14.5 \%$ of our study population used levothyroxine, and no subject had been diagnosed with thyroid cancer, we considered these patients to be under treatment for primary hypothyroidism. We observed a high prevalence of undertreated patients, but some authors have shown that about $60 \%$ of patients receiving levothyroxine have euthyroidism [13], similar to the results of the present study.

Subjects undergoing levothyroxine replacement with elevated serum TSH levels tended to show worse performance in ADLs than those adequately treated, although this difference was not significant. These data must be interpreted with caution, given the small sample size. These endpoints appeared to be similar in patients with euthyroidism and adequately treated subjects.

We found no significant association between cognitive and thyroid functions in the present study. Memory alterations and reduced cognitive performance are the most common issues in patients with $\mathrm{OH}$ [1]. Some trials have shown improved cognitive function after levothyroxine replacement, but results have not been uniform, and no age-stratified analysis has demonstrated such an effect [26, 27, 29-31]. Studies of correlations among age, subclinical hypothyroidism, and cognition are difficult to perform because cognitive function declines with age. Most longitudinal studies have failed to demonstrate that elevated serum TSH is related to cognitive effects, which may be related to methodological issues [26]. MMSE, the screening tool for cognitive decline used in this study, is not a very sensitive test, besides it is largely employed in the literature despite this limitation. Additional studies using more specific methodologies to examine cognitive function in elderly patients with subclinical hypothyroidism, and the practical repercussions of any such association are needed. Given the relationship between depression and cognition, some studies have demonstrated an association between minimal elevations on serum TSH and depressive symptoms in elderly individuals [53, 54], but other studies using several screening tools have found no such association $[55,56]$. In the present study, no difference in depressive symptoms was observed between patients with euthyroidism and hypothyroidism, irrespective to age.

Limitations of this study include the characteristics of the population, which was highly selected in an outpatient setting, resulting in a high prevalence of comorbidities, use of multiple medications, and low functional status. Population-based studies are needed to corroborate our findings. Some losses of study subjects occurred and were related to their dependence on proxies or caregivers to escort them to the clinic for complete evaluation. This last limitation is also associated with the small number and the characteristics of a convenient selected sample, which might influence some negative results in the study. The well-known limitations of cross-sectional studies' results also apply to the present study. Prospective studies should be conducted to more thoroughly explore our findings. Few studies have assessed thyroid status in elderly, especially very elderly, individuals, who represent the most rapidly growing age group worldwide. Better knowledge of this and other aspects in the very elderly population is urgently needed.

\section{Conclusions}

Subclinical hypothyroidism does not impact functional performance in the elderly population as a whole. However, serum TSH above the reference range may be associated with better functional status in subjects aged $\geq 85$ years. More thorough studies are needed to assess these findings and their impact on hospitalization and mortality in this specific population.

Conflict of interest All the authors have no conflict of interest to declare.

Open Access This article is distributed under the terms of the Creative Commons Attribution License which permits any use, distribution, and reproduction in any medium, provided the original author(s) and the source are credited.

\section{References}

1. J.W. Smith, A.T. Evans, B. Costall, J.W. Smythe, Thyroid hormones, brain function and cognition: a brief review. Neurosci. Biobehav. Rev. 26, 45-60 (2002) 
2. P. Kaminsky, M. Klein, M. Duc, Hypothyroid myopathyphysiopathological approach. Ann. Endocrinol. 53, 125-132 (1992)

3. A. Moura Neto, M.C. Parisi, M.A. Tambascia, E.J. Pavin, S.M. Alegre, D.E. Zantut-Wittmann, Relationship of thyroid hormone levels and cardiovascular events in patients with type 2 diabetes. Endocrine (2013). doi:10.1007/512020-0139938-6

4. J. Klein, K. Ojamaa, Thyroid hormone and the cardiovascular system. N. Engl. J. Med. 344, 501-509 (2001)

5. G. Vitale, L.M. Fatti, S. Prolo, A. Girola, M. Caraglia, M. Marra, A. Abbruzzese, G. Gerli, D. Mari, Screening for hypothyroidism in older hospitalized patients with anemia: a new insight into an old disease. J. Am. Geriatr. Soc. 58, 1825-1827 (2010)

6. G. Vitale, S. Salvioli, C. Franceschi, Oxidative stress and the ageing endocrine system. Nat. Rev. Endocrinol. 9, 228-240 (2013)

7. G.P. Bianchi, V. Zaccheroni, E. Solaroli, F. Vescini, R. Cerutti, M. Zoli, G. Marchesini, Health-related quality of life in patients with thyroid disorders. Qual. Life Res. 13, 45-54 (2004)

8. S. Gulseren, L. Gulseren, Z. Hekimsoy, C. Ozen, B. Tokatliogen, Depression, anxiety, health-related quality of life, and disability in patients with overt and subclinical thyroid function. Arch. Med. Res. 37, 133-139 (2006)

9. E.M. Simonsick, A.B. Newman, L. Ferrucci, S. Satterfield, T.B. Harris, N. Rodondi, Subclinical hypothyroidism and functional mobility in older adults. Arch. Intern. Med. 169, 2011-2017 (2009)

10. W.M.G. Tunbridge, D.C. Evered, R. Hall, D. Appleton, M. Brewis, F. Clark, The spectrum of thyroid disease in a community: the Whickham survey. Clin. Endocrinol. 7, 481-493 (1977)

11. N. Bagchi, T.R. Brown, R.F. Parish, Thyroid dysfunction in adults over age 55 years: a study in an urban US community. Arch. Intern. Med. 150, 785-786 (1990)

12. J.V. Parle, J.A. Franklyn, K.W. Cross, S.C. Jones, M.C. Sheppard, Prevalence and follow-up of abnormal thyrotrophin (TSH) concentrations in the elderly in the United Kingdom. Clin. Endocrinol. 34, 77-83 (1991)

13. G.J. Canaris, N.R. Manowitz, G. Mayor, C. Ridgway, The colorado thyroid disease prevalence study. Arch. Intern. Med. 160, 526-534 (2000)

14. R. Sichieri, J. Baima, T. Marante, M.T.L. Vasconcellos, A.S. Moura, M. Vaismam, Low prevalence of hypothyroidism among black and Mulatto people in a population-based study of Brazilian women. Clin. Endocrinol. 66, 803-807 (2007)

15. B. Biondi, D.S. Cooper, The clinical significance of subclinical thyroid dysfunction. Endocr. Rev. 29, 76-131 (2008)

16. G. Atzmon, N. Barzilai, J.G. Hollowell, M.I. Surks, I. Gabriely, Extreme longevity is associated with increased serum thyrotropin. J. Clin. Endocrinol. Metab. 94, 1251-1254 (2009)

17. M.I. Surks, J.G. Hollowell, Age-specific distribution of serum thyrotropin and antithyroid antibodies in the US population: implications for the prevalence of subclinical hypothyroidism. J. Clin. Endocrinol. Metab. 92, 4575-4582 (2007)

18. I.M. Benseñor, A.C. Goulart, P.A. Lotufo, P.R. Menezes, M. Scazufca, Prevalence of thyroid disorders among older people: results from the São Paulo Ageing and Health Study. Cad. Saude Publica. 27, 155-161 (2011)

19. D. Maugeri, M.S. Russo, F. Di Stefano, G. Receputo, D. Rosso, R. Rapisarda, Thyroid function in healthy centenarians. Arch. Geront. Geriatr. 25, 211-217 (1997)

20. D.S. Cooper, B. Biondi, Subclinical thyroid disease. Lancet 379, 1142-1154 (2012)

21. J.R. Garber, R.H. Cobin, H. Gharib, J.V. Hennessey, I. Klein, J.I. Mechanick, R. Pessah-Pollack, P.A. Singer, K.A. Woeber, Clinical practice guidelines for hypothyroidism in adults: cosponsored by the American Association of Clinical Endocrinologists and the American Thyroid Association. Thyroid 22, 1200-1236 (2012)

22. J.A. Sgarbi, P.F.S. Teixeira, L.M.Z. Maciel, G.M.F.S. Mazeto, M. Vaisman, R.M. Montenegro Jr, L.S. Ward, The Brazilian consensus for the clinical approach and treatment of subclinical hypothyroidism in adults: recommendations of the thyroid Department of the Brazilian Society of Endocrinology and Metabolism. Arq. Bras. Endocrinol. Metabol. 57, 166-183 (2013)

23. G. Atzmon, N. Barzilai, M.I. Surks, I. Gabriely, Genetic predisposition to elevated serum thyrotropin is associated with exceptional longevity. J. Clin. Endocrinol. Metab. 94, 4768-4775 (2009)

24. L.L. Somwaru, C.M. Rariy, A.M. Arnold, A.R. Cappola, The natural history of subclinical hypothyroidism in the elderly: the Cardiovascular Health Study. J. Clin. Endocrinol. Metab. 97, 1962-1969 (2012)

25. J. Parle, P. Maisonneuve, M. Sheppard, P. Boyle, J. Franklyn, Prediction of all-cause and cardiovascular mortality in elderly people from one low serum thyrotropin result: a 10-year Cohort Study. Lancet 358, 861-865 (2001)

26. M.H. Samuels, Cognitive function in subclinical hypothyroidism. J. Clin. Endocrinol. Metab. 95, 3611-3613 (2010)

27. J. Parle, L. Roberts, S. Wilson, H. Pattison, A. Roalfe, M.S. Haque, A randomized controlled trial of the effect of thyroxine replacement on cognitive function in community-living elderly subjects with subclinical hypothyroidism: the Birmingham Elderly Thyroid Study. J. Clin. Endocrinol. Metab. 95, 3623-3632 (2010)

28. G. Ceresini, F. Lauretani, M. Maggio, G.P. Ceda, S. Morganti, E. Usberti, C. Chezzi, R. Valcavi, S. Bandinelli, J.M. Guralnik, A.R. Cappola, G. Valenti, L. Ferrucci, Thyroid function abnormalities and cognitive impairment in elderly people: results of the Invecchiare in Chianti Study. J. Am. Geriatr. Soc. 57, 89-93 (2009)

29. R. Jorde, K. Waterloo, H. Storhaug, A. Nyrnes, J. Sundsfjord, T.G. Jenssen, Neuropsychological function and symptoms in subjects with subclinical hypothyroidism and the effect of thyroxine treatment. J. Clin. Endocrinol. Metab. 91, 145-153 (2006)

30. Y.J. Park, E.J. Lee, Y.J. Lee, S.H. Choi, J.H. Park, S.B. Lee, Subclinical hypothyroidism ( $\mathrm{SCH})$ is not associated with metabolic derangement, cognitive impairment, depression or poor quality of life (QoL) in elderly subjects. Arch. Geront. Geriatr. 50, e68-e73 (2010)

31. I. Bensenor, Screening for thyroid disorders in symptomatic adults from Brazilian populations. São Paulo Med. J. 120, 146-151 (2002)

32. J. Gussekloo, E.V. Exel, A.J. Craen, A.E. Meinders, M. Frölich, R.G.J. Westendorp, Thyroid status, disability and cognitive function, and survival in old age. JAMA 292, 2591-2599 (2004)

33. G. Pasqualetti, S. Tognini, A. Polini, N. Caraccio, F. Monzani, Is subclinical hypothyroidism a cardiovascular risk factor in the elderly? J. Clin. Endocrinol. Metab. (2012). doi:10.1210/jc.20123818

34. K.A. Hyland, A.M. Arnold, J.S. Lee, A.R. Cappola, Persistent subclinical hypothyroidism and cardiovascular risk in the elderly: the Cardiovascular Health Study. J. Clin. Endocrinol. Metab. 98, 533-540 (2013)

35. Y.A.O. Duarte, C.L. Andrade, M.L. Lebrão, Katz index on elderly functionality evaluation. Rev. Esc. Enferm. USP. 41, 317-325 (2007)

36. S. Katz, Studies of illness in the aged-the index of ADL: a standardized measure of biological and psychosocial function. JAMA 185, 914-919 (1963)

37. V.T.S. Lino, S.R.M. Pereira, L.A.B. Camacho, S.T.R. Filho, S. Buksman, Cross-cultural adaptation of the independence in activities of daily living Index (Katz Index). Cad. Saude Publica. 24, 104-112 (2008) 
38. B. Bruce, J.F. Fries, The Stanford Health Assessment Questionnaire: a review of its history, issues, progress, and documentation. J. Rheumatol. 30, 167-178 (2003)

39. M.B. Ferraz, L.M. Oliveira, P.M. Araujo, E. Atra, P. Tugwell, Crosscultural reliability of the physical ability dimension of the Health Assessment Questionnaire. J. Rheumatol. 17, 813-817 (1990)

40. J.F. Fries, P.W. Spit, D.Y. Young, The dimensions of health outcomes: the Health Assessment Questionnaire, disability and pain scales. J. Rheumatol. 95, 789-793 (1982)

41. C.E.H. Siegert, L.J. Vleming, J.P. Vandernbroycke, A. Cats, Measurement of disability in Dutch rheumatoid arthritis patients. Clin. Rheumatol. 3, 305-309 (1984)

42. M.F. Folstein, S.E. Foltein, P.R. Mchugh, A practical method for grading the cognitive state of patients for the clinician. J. Psychiatr. Res. 12, 189-198 (1975)

43. R.A. Lourenço, R.P. Veras, Mini-Mental State Examination: psychometric characteristics in elderly outpatients. Rev. Saude Publica 40, 712-719 (2006)

44. J.A. Yesavage, T.L. Brink, T.L. Rose, O. Lum, V. Huang, M. Adey, Development and validation of a geriatric depression screening scale: a preliminary report. J. Psychiatr. Res. 17, 37-49 (1983)

45. O.P. Almeida, S.A. Almeida, Confiabilidade da versão brasileira da Escala de Depressão em Geriatria (GDS) versão reduzida. Arq. Neuropsiquiatr. 57, 421-426 (1999)

46. G.S. Alexopoulos, R.C. Abrams, R.C. Young, C.A. Shamolan, Cornell scale for depression in Dementia. Biol. Psychiatry 23, 271-284 (1988)

47. M.T. Carthery-Goulart, R. Areza-Fegyveres, R.R. Schultz, I. Okamoto, P. Caramelli, P.H.F. Bertolucci, Versão Brasileira da Escala cornell de depressão em Demência (cornell depression scale in Dementia). Arq. de Neuropsiquiatr. 65, 912-915 (2007)
48. S. Razvi, A. Shakoor, M. Vanderpump, J.U. Weaver, S.H. Pearce, The influence of age on the relationship between subclinical hypothyroidism and ischemic heart disease: a metaanalysis. J. Clin. Endocrinol. Metab. 93, 2998-3007 (2008)

49. A.W. Van den Beld, T.J. Visser, R.A. Feelders, D.E. Grobbee, S.W. Lamberts, Thyroid hormone concentrations, disease, physical function, and mortality in elderly men. J. Clin. Endocrinol. Metab. 90, 6403-6409 (2005)

50. A.P. Bremner, P. Feddema, P.J. Leedman, S.J. Brown, J.P. Beilby, E.M. Lim, S.G. Wilson, P.C. O'Leary, J.P. Walsh, Agerelated changes in thyroid function: a longitudinal study of a community-based cohort. J. Clin. Endocrinol. Metab. 97, 1554-1562 (2012)

51. R.P. Veras, Population aging today: demands, challenges and innovations. Rev. Saude Publica 43, 548-554 (2009)

52. J.F. Fries, Aging, natural death and the compression of morbidity. New Engl. J. Med. 303, 130-135 (1980)

53. S. Razvi, L. Ingoe, G. Keeka, C. Oates, C. McMillan, J.U. Weaver, The beneficial effect of L-thyroxine on cardiovascular risk factors, endothelial function, and quality of life in subclinical hypothyroidism: randomized, crossover trial. J. Clin. Endocrinol. Metab. 92, 1715-1723 (2007)

54. J.M. Guimarães, C. de Souza Lopes, J. Baima, R. Sichieri, Depression symptoms and hypothyroidism in a population-based study of middle-aged Brazilian women. J. Affect. Disord. 117, 120-123 (2009)

55. A. Engum, T. Bjøro, A. Mykletun, A.A. Dahl, An association between depression, anxiety and thyroid function-a clinical fact or an artefact? Acta Psychiatr. Scand. 106, 27-34 (2002)

56. R.T. Joffe, E.N. Pearce, J.V. Hennessey, J.J. Ryan, R.A. Stern, Subclinical hypothyroidism, mood, and cognition in older adults: a review. Int. J. Geriatr. Psychiatry 28, 111-118 (2013) 